\title{
UMA ANÁLISE SISTÊMICA SOCIOTECNOLÓGICA DA ENGENHARIA DE REQUISITOS
}

\author{
A SYSTEMIC, SOCIOTECHNOLOGICAL ANALYSIS OF REQUIREMENTS \\ ENGINEERING
}

\author{
Luís Augusto Machado Moretto \\ Mestrando do Programa de Pós-Graduação em Engenharia e Gestão do Conhecimento \\ Universidade Federal de Santa Catarina \\ Professor do Curso de Informática do SENAI-SC \\ malacma@gmail.com \\ Alessandra Maria Ruiz Galdo \\ Mestre em Ciência da Informação \\ Doutoranda do Programa de Pós-Graduação em Engenharia e Gestão do Conhecimento \\ Universidade Federal de Santa Catarina \\ alessandra.galdo@gmail.com \\ Vinícius Medina Kern \\ Professor do Programa de Pós-Graduação em Engenharia e Gestão do Conhecimento \\ Universidade Federal de Santa Catarina \\ Pesquisador do Instituto Stela \\ $\underline{\text { kern@egc.ufsc.br }}$
}

\begin{abstract}
RESUMO
A engenharia de requisitos, etapa inicial da construção de sistemas de informação, requer intenso intercâmbio de informação entre profissionais de tecnologias da informação e comunicação, e peritos no negócio. A teoria e a prática comum da engenharia de requisitos, no entanto, são tecnocêntricas e caracterizadas por dificuldade de comunicação. A literatura recente aborda a concepção de sistemas sociotecnológicos, cujas propriedades emergem por meio da colaboração dinâmica entre pessoas e agentes artificiais. Este artigo apresenta um modelo sistêmico sociotecnológico da engenharia de requisitos com base no modelo CESM de Mario Bunge, segundo o qual todo sistema concreto pode ser representado segundo seus componentes, ambiente, estrutura e mecanismo. Entre os componentes, estão os peritos no negócio, profissionais de TIC, agentes artificiais e os objetos da colaboração dinâmica - modelo de domínio e requisitos. O mecanismo é essencialmente um processo de comunicação, com intercâmbio de informações e compartilhamento de conhecimento. Essa descrição presta-se à compreensão sistêmica da engenharia de requisitos, visando à intervenção para aliviar os problemas comunicacionais do processo e promover a emergência de requisitos que representem as reais necessidades do sistema.
\end{abstract}

PALAVRAS-CHAVE: Sistemas de informação. Sistemas sociotecnológicos. Engenharia de requisitos. Compartilhamento do conhecimento. Visão sistêmica.

\section{INTRODUÇÃO}

McGee e Prusak (1994) afirmam que, na economia da informação, o diferencial competitivo das organizações está na sua capacidade de adquirir, tratar, interpretar e utilizar a informação de forma eficaz. Os sistemas de informação são auxiliares neste processo.

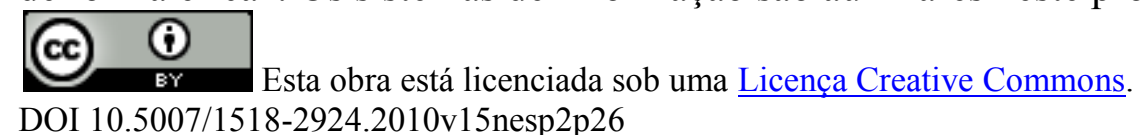

Enc. Bibli: R. Eletr. Bibliotecon. Ci. Inf., Florianópolis, n. esp., 2º sem. 2010. ISSNe 1518-2924. 
Laudon e Laudon (1999, p. 5-6) tratam os sistemas de informação como partes essenciais da organização, com três componentes: "tecnologia, organizações e pessoas", sendo a tecnologia, ou, no caso deste trabalho, a engenharia de requisitos, "o meio pelo qual os dados e as informações são transformados, documentados e validados para o desenvolvimento dos sistemas de informação". A elaboração de um sistema de informação exige comunicação e intensa troca de informações e conhecimentos entre os profissionais de Tecnologias da Informação e Comunicação (TICs) e os profissionais da organização que são usuários ou peritos no negócio enfocado pelo sistema de informação.

$\mathrm{Na}$ concepção de sistemas de informação, a engenharia de requisitos é a etapa inicial, na qual os profissionais de TICs se empenham na compreensão do problema e na especificação do que o sistema deverá fazer - os requisitos desse sistema. É um conjunto de atividades que propiciam a identificação das necessidades do usuário e das expectativas dos clientes ou patrocinadores do sistema de informação, bem como o entendimento do impacto do software na organização e o mapeamento das interações entre os usuários e o software (PRESSMAN, 2005).

A prática comum segue uma abordagem tecnocêntrica, com foco no artefato e não no sistema social que o sistema de informação se propõe a atender. Essa perspectiva torna-se evidente na afirmação de Sommerville (2005), segundo a qual os processos da engenharia de requisitos têm como meta criar e manter documentos de sistema. Kilov e Sack (2009) apontam uma lacuna comunicacional profunda entre os profissionais de TICs e do negócio.

Em uma perspectiva distinta da abordagem tecnocêntrica, entende-se a engenharia de requisitos como um sistema sociotecnológico, um tipo de sistema complexo, autoorganizado, cujo funcionamento depende de uma colaboração dinâmica que envolve pessoas e agentes artificiais. O conceito de sistema sociotecnológico (FUCHS, 2005; ECCLES; GROTH, 2006, 2007) representa um avanço em relação à visão tecnocêntrica dos sistemas de informação que possuem um forte componente computacional.

Apesar desse avanço, a prática da engenharia de requisitos continua tecnocêntrica. Como compreendê-la como sistema sociotecnológico? Este artigo trata de modelar um sistema sociotecnológico da engenharia de requisitos a partir do modelo composition-environmentstructure-mechanism (CESM) de Bunge (2003), segundo o qual qualquer sistema concreto pode ser descrito segundo seus componentes, ambiente, estrutura e mecanismo.

Bunge (1997) propõe regras metodológicas gerais para a pesquisa com visão sistêmica, segundo as quais se deve colocar cada fato social no seu contexto mais amplo, i. e., sistema, para depois descrever a composição, ambiente e estrutura, distinguir outros níveis de sistema e suas relações, procurar por (ou conjeturar) mecanismos da emergência, crescimento, manutenção ou decadência, testar esses mecanismos e, em caso de mau funcionamento, examinar as quatro fontes possíveis (CESM) e buscar reparar o sistema, alterando uma ou mais dessas quatro possíveis fontes. No presente estudo, o sistema é a engenharia de requisitos de sistemas de informação e a delimitação do estudo inclui a descrição de composição, ambiente e estrutura e a conjetura de mecanismos, considerando a possibilidade da intervenção de agentes tecnológicos na colaboração existente entre peritos no negócio e em TICs. 
O artigo discorre, na próxima seção, sobre conceitos fundamentais da engenharia de software, engenharia de requisitos e sistemas sociotecnológicos. A seção três apresenta um modelo sistêmico sociotecnológico da engenharia de requisitos com base na abordagem sistêmica de Bunge (1997). Detalham-se os componentes (pessoas: peritos no negócio e em TICs, agentes artificiais e artefatos: o modelo de domínio e a especificações de requisitos), os itens do ambiente, as principais ligações internas e com o ambiente, e os mecanismos geradores de propriedades emergentes (do todo) do sistema. A seção quatro apresenta conclusões e direções de pesquisa.

\section{FUNDAMENTOS: ENGENHARIA DE SOFTWARE, ENGENHARIA DE REQUISITOS, SISTEMAS SOCIOTECNOLÓGICOS}

Saracevic (1996) explica que a Ciência da Informação (CI) se relaciona às TICs e é uma ciência interdisciplinar em que as dimensões humana e social ultrapassam a dimensão tecnológica. A abordagem sociotecnológica de sistemas de informação, tratada nesse trabalho, contrasta com a visão tecnocêntrica ao compreender os aspectos técnicos e sociais como partes de um mesmo sistema. Na definição de Fuchs (2005), sistemas sociotecnológicos se auto-organizam por meio da colaboração dinâmica entre pessoas e tecnologia ou, nas palavras do autor, entre agentes humanos e artificiais.

Riecken (2006) analisa as linhas de pesquisa da CI e relaciona as TICs entre os cinco principais eixos da área, quais sejam: epistemologia; arquivologia, biblioteconomia e documentação; TICs; necessidade social; gestão de Recursos Humanos. Em relação ao eixo das TICs, Riecken (2006, p. 54) afirma que os temas tratados usualmente se referem principalmente às "aplicações ou modelos, visando à operacionalização em ambientes computacionais" e tem como base a Teoria Geral da Informação, a Cibernética, os Bancos de Dados, a Teoria Geral dos Sistemas, a Ciência da Computação, a Tecnologia de Informação, bem como a Engenharia de Software. Este trabalho apresenta uma visão sistêmica e um modelo sociotecnológico da engenharia de requisitos, uma das áreas da Engenharia de Software, como detalhado a seguir.

\subsection{Engenharia de requisitos}

A engenharia de requisitos é a etapa inicial na construção de sistemas de informação. Sommerville (2005) conceitua os requisitos de software como a descrição dos serviços ofertados pelo sistema e suas restrições operacionais. Os requisitos refletem as necessidades do usuário, auxiliando na resolução de um determinado problema, como buscar informações ou efetuar pedidos para uma organização. A engenharia de requisitos é definida pelas seguintes fases (BOURQUE; DUPUIS, 2004; PRESSMAN, 2005):

a) Elicitação: identificação da origem e coleta dos requisitos pelo profissional de TIC;

b) Análise: resolução de conflitos e ambiguidades entre os requisitos;

c) Especificação: documentação sistemática de um documento eletrônico que possa ser revisado, avaliado e aprovado;

d) Validação: revisão, avaliação da qualidade e aprovação dos requisitos pelos peritos no negócio;

e) Controle de mudanças e rastreabilidade: identificação dos impactos e de quais requisitos são impactados com as mudanças. 
Os requisitos de software são classificados em funcionais e não funcionais. Os requisitos funcionais são descritos como as funções contidas no sistema de informação e suas propriedades. São ainda chamados de capacidades do sistema. Como exemplo de um requisito funcional, cita-se a formatação de um texto, a correção ortográfica e a interpretação de um sinal. Os requisitos não funcionais são definidos em termos de propriedades qualitativas dos sistemas de informação. São sub-classificados em categorias como desempenho, manutenibilidade, segurança, flexibilidade e confiabilidade (BOURQUE; DUPUIS, 2004; PRESSMAN, 2005; SCHNEIDER, 2009).

A comunicação, a troca de informações e o compartilhamento do conhecimento entre os atores envolvidos na engenharia de requisitos causam impacto na qualidade dos processos da engenharia de requisitos. Entre os processos impactados, pode-se citar a validação dos requisitos, verificação dos requisitos no software e a estimativa dos custos (BOURQUE; DUPUIS, 2004).

\subsection{Sistemas sociotecnológicos}

O primeiro grande contraponto à visão tecnocêntrica dos sistemas de informação foi a abordagem sociotécnica, que reconhece a influência mútua entre os subsistemas social e técnico (CHERNS, 1976), porém de forma dualista. Sawyer e Crowston (2004) consideram que a abordagem sociotécnica permanece dividida em dois enfoques, o social e o técnico.

Nas abordagens sobre sistemas de informação, Alter (2003) propõe substituir a noção de "artefato de TI", típico da visão tecnocêntrica, por "sistemas de trabalho dependentes de TI". Os sistemas de informação não são formados apenas por artefatos tecnológicos, mas por pessoas, informação e tecnologia, como conceituam Laudon e Laudon (1999).

Fuchs (2005) vai além da visão sociotécnica e concebe a noção de sistemas sociotecnológicos, sistemas que se auto-organizam por meio da colaboração dinâmica entre agentes humanos e agentes artificiais. A auto-organização do sistema depende de intensa comunicação. Um exemplo de sistema sociotecnológico para o autor é a Internet, na qual a atividade social, a comunicação do tipo muitos-muitos e a interação entre ferramentas tecnológicas e pessoas produzem a auto-organização sistêmica.

A abordagem sociotecnológica permite a concepção de sistemas para a resolução de problemas que podem ser aplicados à questão da comunicação e do compartilhamento do conhecimento na engenharia de requisitos (FUCHS, 2005; ECCLES; GROTH, 2006, 2007). Essa abordagem enfoca os atores do processo e a comunicação tanto entre os peritos em negócio com os profissionais de TICs, como entre pessoas e artefatos tecnológicos, ou entre agentes humanos e agentes artificiais ${ }^{1}$.

Um sistema sociotecnológico pode ser representado por um modelo ontológico. Nesse trabalho, usa-se o conceito de Borst $(1997$, p. 12) para ontologia: "uma especificação formal de uma conceitualização compartilhada". Esse conceito é o mais apropriado, pois, corroborando com Almeida e Bax (2003, p. 9), é uma "definição simples e completa" para a

\footnotetext{
${ }^{1}$ Um agente artificial é uma entidade computacional que atua na resolução de problemas (D'INVERNO; LUCK, 2001).
} 
expressão que vem sendo utilizada por diversas áreas, como a Filosofia, as Ciências da Computação e a Ciência da Informação com significações por vezes distintas.

O compartilhamento de conceitos e conhecimentos é necessário para a eficácia da comunicação entre os profissionais de TICs e os peritos de negócio. Assim, desenvolve-se nesse trabalho um modelo ontológico para representar um sistema sociotecnológico, adaptável a outros sistemas, como aqueles cuja construção depende da comunicação e compartilhamento de conhecimentos entre os profissionais de TICs e os peritos de negócios.

\section{UM MODELO SOCIOTECNOLÓGICO DA ENGENHARIA DE REQUISITOS}

Sayão (2001, p. 90) explica que a ciência trabalha com abstrações da realidade. Considera que os modelos "desempenham um papel importante como recurso metodológico para todas as áreas cujos interesses são os fenômenos relacionados à informação, como a informática e a ciência da informação".

A visão de mundo sistêmica adotada neste trabalho é baseada no modelo $\mathrm{CESM}^{2}$ de Bunge (2003). O autor analisa diversas definições de sistemas e considera a necessidade de um conceito científico abrangente. Considera as partes comuns a qualquer sistema concreto, sejam sistemas naturais, sociais, técnicos ou semióticos. Concebe o modelo CESM, que prevê também os fatores externos ao sistema (ambiente), as ligações entre os componentes do sistema (endoestrutura), bem como entre esses e os itens do ambiente (exoestrutura). $\mathrm{O}$ modelo é representado pela seguinte notação:

$$
\mathrm{m}(\mathrm{s})=\langle\mathrm{C}(\mathrm{s}), \mathrm{E}(\mathrm{s}), \mathrm{S}(\mathrm{s}), \mathrm{M}(\mathrm{s})\rangle
$$

onde:

$\boldsymbol{s}=$ sistema;

$\mathrm{C}(\mathrm{s})=$ Componentes: coleção de todas as partes de $\boldsymbol{s}$;

$\mathrm{E}(\mathrm{s})=$ Ambiente ou entorno: coleção dos elementos não pertencentes a $\boldsymbol{s}$, mas que atuam sobre os componentes e/ou são afetados pela atuação dos componentes de $\boldsymbol{s}$;

$\mathrm{S}(\mathrm{s})=$ Estrutura: conjunto de relações, em particular, vínculos entre os componentes de $s$, ou entre esses e os elementos do entorno $\mathbf{E}(\mathbf{s})$;

$\mathrm{M}(\mathrm{s})=$ Mecanismo: Coleção de processos de $\boldsymbol{s}$ que o fazem comportar-se da maneira peculiar com que se comporta.

Concebe-se um modelo sistêmico, no qual os componentes do sistema são peritos no negócio, os profissionais em TIC, os agentes artificiais, o modelo de domínio e os requisitos - os objetos de colaboração dinâmica entre os atores na engenharia de requisitos (Figura 1).

\footnotetext{
${ }^{2}$ CESM deriva das inicias das partes que compõem o modelo sistêmico. Composition, environment, structure, mechanism . Em português: Componentes, Ambiente, Estrutura, Mecanismo.
} 


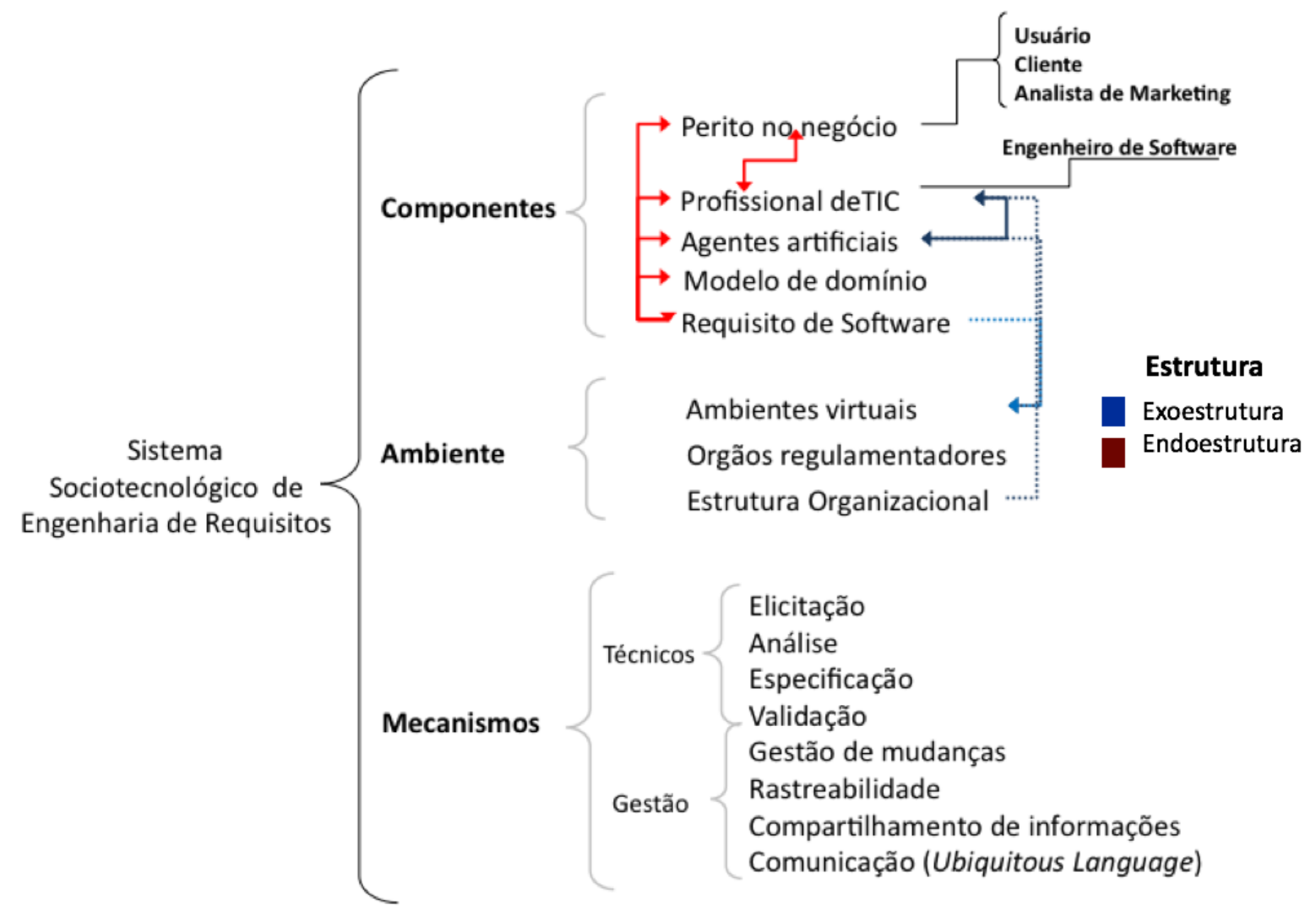

Figura 1: Modelo sociotecnológico da Engenharia de Requisitos

Fonte: Autores

A Figura 1 representa os componentes, ambiente, estrutura e mecanismos de um sistema sociotecnológico, como detalhado a seguir:

\section{a) Componentes}

Bourque e Dupuis (2004) ressaltam a natureza interdisciplinar desta área de conhecimento da engenharia de software e explicitam os seguintes atores:

a) Usuários: usam o software para atender às necessidades em suas atividades fim; grupo heterogêneo com diferentes papéis e conhecimentos;

b) Cliente: comprador ou patrocinador do software;

c) Analista de Marketing: atua como interlocutor entre as demandas do mercado e o engenheiro de software;

Representado pela designação “profissional de TICs, estão os:

d) Engenheiros de software: tem como função compreender as demandas do negócio, e a partir das necessidades do cliente, criar e manter os requisitos de sistema.

Assim, a expressão "perito no negócio" designa os profissionais responsáveis por transferir as informações e o conhecimento sobre as necessidades do usuário e do negócio ao profissional de TIC. A expressão "profissionais de TIC" representa os responsáveis pelo desenvolvimento 
de sistemas. Estas designações são amplamente utilizadas na literatura e foram adotadas a partir de Kilov e Sack (2009), que propõem a criação de um modelo de domínio do conhecimento compartilhado para mitigar a lacuna comunicacional entre os atores.

Segundo Evans (2003), um modelo de domínio do conhecimento não é um diagrama em especial. Representa a ideia que o diagrama destina-se a compartilhar. Não é apenas o conhecimento do perito no negócio, é uma abstração rigorosamente organizada e seletiva de conhecimento. É o coração da abordagem Domain Driven Design (DDD), auxiliando na captura dos conceitos e das responsabilidades do software (HAYWOOD, 2009). Este componente favorece o mecanismo de comunicação por meio da ubiquitous language $e^{3}$, a "linguagem onipresente" que permite falar sobre o domínio do conhecimento de forma inteligível para peritos em TICs e no negócio.

Eccles e Groth (2006) afirmam que, nos sistemas sociotecnológicos, os agentes humanos. juntamente com os agentes artificiais, possuem capacidades de resolução de problemas que são superiores às capacidades individuais. Os agentes artificiais formam sistemas distribuídos que colaboram entre si para a resolução de um determinado problema. Apresentam propriedades como: reatividade, característica de responder em tempo útil às mudanças do ambiente; continuidade de percepção e atuação no ambiente; orientação a objetos, não se limitando a interagir apenas com o ambiente; possuem um estado e realizam operações dentro de seu contexto. São providos de graus de autonomia visto como a motivação do agente (D'INVERNO; LUCK, 2001).

Para Sommerville (2005), os requisitos são descritos em linguagem natural e a partir de diagramas. Representam os serviços a executar e as restrições que o software deve atender. Expressam as necessidades do produto de software para solucionar um problema do mundo real. Podem ser explicitados em alto nível de abstração como um serviço ou detalhados formalmente através da Unified Modeling Language (UML) ${ }^{4}$ (OBJECT MANAGEMENT GROUP, 2009).

Os requisitos podem assumir uma representação formal ou semi-formal. Como exemplo de notação formal a UML provê 14 diagramas técnicos para análise e projeto orientado a objetos, possibilitando o rigor técnico na especificação dos requisitos de software (OBJECT MANAGEMENT GROUP, 2009; AMBLER, 2004).

\section{b) Ambiente}

Para Schneider (2009) o principal motivo para a implantação de práticas de gestão do conhecimento nas organizações é o fato de uma organização inteligente reagir com maior agilidade, precisão e qualidade às demandas dos clientes e do mercado. Morgan (1996, p. 84) afirma que "as organizações são sistemas de processamento de informações capazes de aprender a aprender".

A partir da abordagem da gestão do conhecimento, descrevem-se possíveis interferências do ambiente no processo de Engenharia de requisitos, como: cultura e poder; órgãos

\footnotetext{
${ }^{3}$ Segundo Evans (2003), a ubiquitous language inclui termos para discutir as regras explícitas no modelo de domínio. É utilizada pelos componentes humanos do sistema para descrever os artefatos, tarefas e funcionalidades do sistema de informação.

4 É uma linguagem de modelagem unificada para representação de diagramas técnicos dos sistemas de informação (OBJECT MANAGEMENT GROUP, 2009).

Enc. Bibli: R. Eletr. Bibliotecon. Ci. Inf., Florianópolis, n. esp., 2º sem. 2010. ISSNe 1518-2924.
} 
regulamentadores; estrutura organizacional e os ambientes virtuais. Segundo Schreiber (2000), a cultura e poder são partes do ambiente implícitas no conhecimento organizacional. Questões como trabalho e comunicação estão diretamente relacionadas aos aspectos sociais e interpessoais, como as competências e as redes de relacionamento informais.

Bourque e Dupuis (2004) destacam interferências do ambiente externo principalmente em setores econômicos, como o bancário, imobiliário e de transportes, que possuem regras para regulamentar suas atividades. Este fator externo à organização influencia na engenharia de requisitos e determina as regras do negócio, os requisitos funcionais e não funcionais do sistema.

Uma estrutura organizacional burocrática e centralizadora pode reprimir a criação de novos conhecimentos. Isto significa que quanto menos hierárquica a estrutura organizacional, maior o grau de compartilhamento do conhecimento entre as pessoas (SHARRATT; USORO, 2003).

Como partes essenciais do ambiente sociotecnológico de engenharia de requisitos encontramse os ambientes virtuais, que podem ter a função de repositórios de conhecimento ou plataformas colaborativas. Shull et al. (2004) destacam a utilidade dos portais Web para a externalização e internalização do conhecimento. Como exemplo de ambientes virtuais e plataformas colaborativas, os wikis têm sido empregados para facilitar a comunicação e gerenciar equipes devido à arquitetura colaborativa, comunicação do tipo muitos-muitos, interface intuitiva e capacidade de extensão (CAVALCANTI; NEPOMUCENO, 2007; FARENHORST; Van VLIET, 2009).

Decker et al. (2005) destacam os wikis como ambientes leves e flexíveis que podem ser utilizados para troca e reuso de artefatos entre os projetos de software, tornando-se um tipo de memória organizacional. Facilitam a comunicação por meio de um conjunto de funcionalidades.

\section{c) Estrutura}

A estrutura do sistema é classificada em endoestrutura, delimitando as interações entre os componentes, e em exoestrutura, formada pelas interações entre os componentes e os itens do ambiente (BUNGE, 2003). Na endoestrutura, o sistema segue uma abordagem em equipe. Eccles e Groth (2006) descrevem que nesta abordagem os agentes artificiais são considerados integrantes da equipe. São capazes de trabalhar de forma independente e, ao mesmo tempo, interagir na resolução de problemas.

As principais ligações do sistema sociotecnológico de Engenharia de requisitos são enunciados evidenciando os componentes e o ambiente, as relações com o perito de negócios, profissional de TICs, os requisitos do sistema e os agentes artificiais e suas funções no sistema de informações, conforme o Quadro 1. 


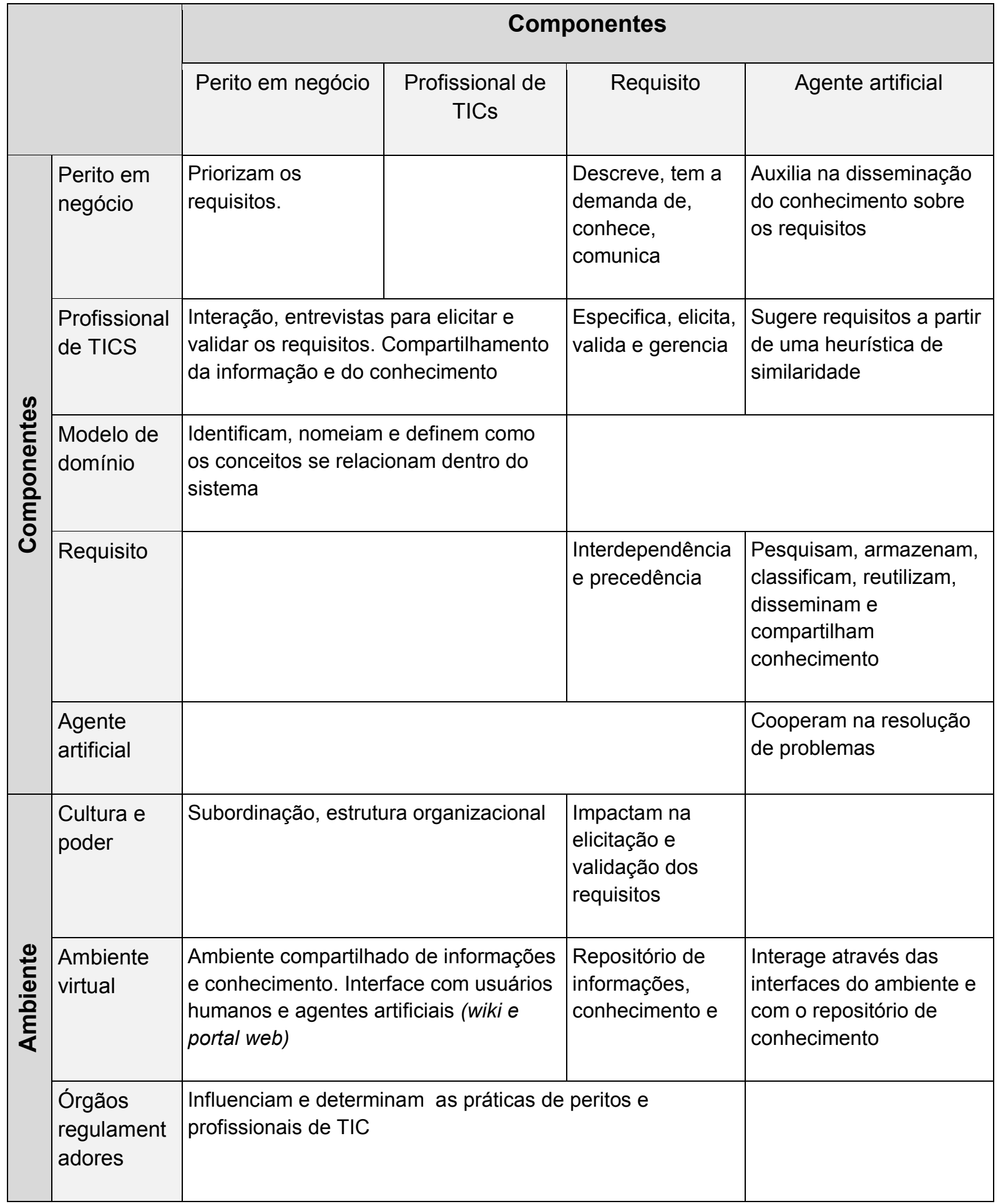

Quadro 1: Ligações do sistema sociotecnológico de Engenharia de Requisitos

Fonte: Autores

Os peritos de TICs identificam e formalizam os requisitos; aplicam técnicas, como entrevistas estruturadas ou semi-estruturadas; análise de protocolos; ordenação de conceitos e grades de repertórios para identificar os requisitos (SCHREIBER, 2000). 
As interações na exoestrutura do sistema sociotecnológico ocorrem entre os itens do ambiente e os componentes (BUNGE, 2003). Podem ocorrer de forma síncrona em salas de conversação e videoconferências ou assíncrona como e-mail e fóruns.

Decker et al. (2005) relatam que os wikis podem ser vistos como memórias organizacionais e fábricas de experiências. Facilitam a comunicação e flexibilizam sua coordenação conforme a cultura, o poder e as estruturas organizacionais.

Os agentes artificiais interagem com os peritos em negócio e profissionais de TIC por meio do ambiente virtual. Auxiliam na busca, classificação, reutilização e compartilhamento dos requisitos. D'Inverno e Luck (2001) afirmam que as ações desempenhadas pelos agentes dependem do contexto. Cada agente desempenha um conjunto de ações conforme suas capacidades e propósitos. Atua em colaboração dinâmica com as pessoas por meio do requisito de software, o artefato essencial do sistema de informação.

\section{d) Mecanismos}

Os mecanismos são os processos que levam a um determinado comportamento sistêmico. São característicos de sistemas concretos e trazem mudanças para o todo, fazendo emergir propriedades e outros processos (BUNGE, 2003). Os mecanismos da engenharia de requisitos podem ser classificados em mecanismos técnicos e em mecanismos de gerência. Os mecanismos técnicos são: elicitação, análise, especificação e a validação. Os mecanismos de gestão são: a gestão de mudanças, rastreabilidade, a construção e uso da ubiquitous language e o compartilhamento das informações e do conhecimento.

A elicitação de requisitos é o primeiro estágio para compreensão das demandas do sistema de informação a ser projetado (SOMMERVILLE, 2005). É responsabilidade dos profissionais de TICs efetuar a interlocução entre as necessidades do perito no negócio e as especificações técnicas dos sistemas de informação (BOURQUE; DUPUIS, 2004).

A análise tem como objetivo detectar e resolver problemas e conflitos entre os requisitos; mapear o escopo do sistema de informação; e a elaboração dos requisitos de sistema para derivar os requisitos de software (BOURQUE; DUPUIS, 2004). Nesse processo, os profissionais de TICs precisam compreender as necessidades do negócio. Requer conhecimento, experiência no domínio de conhecimento do negócio e habilidade de comunicação interdisciplinar (SCHNEIDER, 2009).

Segundo Pressman (2005), no contexto dos sistemas de informação, a especificação dos requisitos pode ser: um documento escrito; um diagrama da UML um modelo formal matemático; um conjunto de casos de uso ou qualquer combinação destes. É durante a especificação que os profissionais de TICs mapeiam os requisitos funcionais, não funcionais e as fronteiras com outros sistemas de informação (SOMMERVILLE, 2005).

A validação dos requisitos consiste em identificar se a especificação do sistema de informação reflete as necessidades explicitadas pelo perito no negócio (SCHNEIDER, 2009). Pressman (2005) explicita que a validação dos requisitos tem como objetivo identificar se todos os requisitos foram representados de forma não ambígua, consistente, sem omissões para detecção e correção dos erros. 
A gestão de mudanças começa com a tarefa de identificar cada um dos requisitos de software. Durante o processo, é atribuído um identificador único aos requisitos para possibilitar a construção de uma matriz de rastreabilidade. Esta matriz se dá em função dos casos de uso e requisitos de software. Tem como propósito mapear os impactos das mudanças no processo de desenvolvimento dos sistemas de informação (PRESSMAN, 2005).

A comunicação por meio da ubiquitous language demanda que os agentes humanos trabalhem para compreender o problema de domínio e que os peritos no negócio sejam precisos em suas nomenclaturas e na descrição dos requisitos. Com isto, os peritos no negócio e profissionais de TIC devem utilizar um vocabulário comum. O resultado é a criação de um modelo de domínio que remete aos principais conceitos do domínio de negócio. Um modelo de domínio único, provendo um vocabulário compartilhado para o sistema (HAYWOOD, 2009).

Para que profissionais de TICs e os peritos em negócio compartilhem informações e conhecimento, é necessário que se estabeleça uma relação de confiança (WANG; NOE, 2010). Ming-Ji; Shiu-Wan; Chih-Jou (2009) definem confiança como a intenção e o comportamento entre os membros do time.

O senso de comunidade nos ambientes virtuais é definido como o sentimento de pertencimento ao grupo. É perspectiva do conhecimento como um bem público que pertence e é mantido pela comunidade. Leva à noção de que quanto maior o senso de comunidade nos ambientes virtuais, melhor será a participação no compartilhamento do conhecimento (SHARRATT; USORO, 2003).

Para que as pessoas compartilhem informações e conhecimento, precisam estar motivadas. A literatura sugere que incentivos e recompensas influenciam no compartilhamento do conhecimento. Quando o compartilhamento das informações e do conhecimento é explicitamente relacionado ao crescimento na carreira, este será positivamente influenciado (SHARRATT; USORO, 2003; WANG; NOE, 2009).

Para favorecer a sinergiana na auto-organização do sistema, é essencial a existência de um processo de coordenação. Eccles e Groth (2006) propõem a metáfora dos seres vivos para conceber a coordenação, comunicação e colaboração entre as pessoas e os agentes artificiais. Para habilitar esse mecanismo no presente sistema, adotou-se, na endoestrutura, a perspectiva de equipes para facilitar a colaboração entre os agentes artificiais e as pessoas em torno do requisito de software, cujo cumprimento é essencial para o sistema de informação.

\section{CONSIDERAÇÕES FINAIS}

A principal contribuição deste artigo é a descrição de um modelo sistêmico sociotecnológico da engenharia de requisitos, etapa inicial da engenharia de software, disciplina fundamental na construção de sistemas de informação. O artigo parte da constatação do caráter tecnocêntrico da teoria e da prática da engenharia de requisitos e apropria-se do conceito de sistema sociotecnológico de Fuchs (2005) para assim conceber um modelo da engenharia de requisitos.

Essa concepção adota o modelo CESM de Bunge (2003), de forma a sistematizar a descrição da engenharia de requisitos como sistema sociotecnológico, a partir de um modelo baseado 
numa ontologia, como uma conceitualização compartilhada, explícita e formal. No modelo bungeano, os componentes e os itens do ambiente são caracterizados por elementos concretos e palpáveis. A estrutura, embora concreta, é menos palpável, pois as ligações podem ser detectadas, mas nem sempre de forma direta (pelos sentidos externos, como são detectados comumente os componentes).

O mecanismo é usualmente invisível e deve ser conjeturado, sujeito a verificação empírica. Ainda que isso seja uma limitação da modelagem CESM, o mecanismo é o que sustenta o funcionamento do sistema, pois se refere ao processo de comunicação e intercâmbio de informações (BUNGE, 2003). Este artigo visou à descrição do sistema sociotecnológico, sem avançar na proposição e teste de agentes que possam alterar essa dinâmica de comunicação, que é tema de trabalhos futuros.

Ao conceber a engenharia de requisitos como sistema sociotecnológico, admite-se um novo conceito de "funcionamento" do sistema. Enquanto na abordagem tecnocêntrica da engenharia de requisitos basta que profissionais de TICs capturem o enunciado de requisitos no sistema de informação a partir da interação com os peritos no negócio e obtenham sua validação (usualmente representada pela assinatura do perito responsável no documento de especificação de requisitos) na abordagem sociotecnológica da engenharia de requisitos, o sistema só "funciona" se o mecanismo ou a comunicação e compartilhamento de informações for efetivo.

No caso da engenharia de requisitos, o principal mecanismo é o compartilhamento de conhecimento entre os profissionais de TICs e os peritos no negócio. A visão sociotecnológica permite voltar a atenção para a efetividade desse mecanismo. Em vez de verificar o cumprimento de requisitos, trata-se de verificar a efetividade do processo de compartilhamento de informações e conhecimento.

A análise das ligações entre os componentes do sistema (inexistente na engenharia de requisitos tecnocêntrica) permite lançar luz sobre os problemas de comunicação entre o sistema social, campo de trabalho dos peritos no negócio, e o sistema técnico, foco usual da atenção dos profissionais de TICs. Espera-se, com isso, proporcionar possibilidades de intervenção para aliviar os problemas comunicacionais do processo e contribuir, dessa forma, para melhor conceber e construir sistemas de informação.

\section{REFERÊNCIAS}

ALMEIDA, M. B.; BAX, M. P. Uma visão geral sobre ontologias: pesquisa sobre definições, tipos, aplicações, métodos de avaliação e de construção. Ciência da Informação, Brasília, v. 32, n. 3, p. 7-20, set./dez. 2003.

ALTER, S. 18 reasons why IT-reliant work systems should replace "the IT artifact" as the core subject matter of the IS field. Communications of the Association for Information Systems, Omaha, v. 12, n.1, p. 366-395, 2003.

AMBLER, S. W. The object primer: agile modeling-driven development with UML 2.0. 3. ed. Cambridge: Cambridge University Press, 2004. 545 p. 
BORST, W. N. Construction of engineering ontologies. 1997. $243 \mathrm{f}$. Tese (Doutorado em Sistemas de Informação e Conhecimento)-Centro de Telemática e Tecnologias da Informação, Escola Holandesa de Graduação em Sistemas de Informação e Conhecimento, Universidade de Twente, Enschede, 1997.

BOURQUE, P.; DUPUIS, R. (Eds.). SWEBOK: guide to software engineering body of knowledge. Los Alamitos: Ieee Computer Society, 2004. 204 p. ISBN 0-7695-2330-7.

BUNGE, M. Mechanism and explanation. Philosophy of the Social Sciences, v. 27, n. 4, p. 410-465, 1997.

BUNGE, M. Emergencia y convergência: novedad cualitativa y unidad del conocimiento. Barcelona: Gedisa Editorial, 2003.

CAVALCANTI, M.; NEPOMUCENO, C. O conhecimento em rede. Rio de Janeiro: Elsevier, 2007.

CHERNS, A. The principles of sociotechnical design. Human Relations, Londres, v. 29, n. 8, p. 783-792, 1976.

DECKER B. et al. Self-organized reuse of software engineering knowledge supported by semantic wikis. In: WORKSHOP ON SEMANTIC WEB ENABLED SOFTWARE ENGINEERING; INTERNATIONAL SEMANTIC WEB CONFERENCE, 4.; 2005, Galway. Anais... Galway: PKM, 2005. p. 411-414.

D'INVERNO, M.; LUCK, M. Understanding agent systems. Nova York: Springer Verlang Berlin, 2001. 191 p. ISBN:3-540-41975-6.

ECCLES, D. W.; GROTH, P. T. Agent coordination and communication in sociotechnological systems: design and measurement issues. Interacting with Computers, Londres, v. 18, n. 6, p. 1170-1185, dez. 2006.

ECCLES, D. W.; GROTH, P. T. Wolves, bees, and football: enhancing coordination in sociotechnological problem solving systems through the study of human and animal groups. Computers in Human Behavior, Amsterdam, v. 23, n. 6, p. 2778-2790, nov. 2007.

EVANS, Eric. Domain-driven design: tackling complexity in the heart of software. Massachusetts: Addison Wesley, 2003.

FARENHORST, R.; Van VLIET, H. Undestanding how to support architects in sharing knowledge. In: INTERNATIONAL CONFERENCE ON SOFTWARE ENGINEERING, 9., 2009, Vancouver. Anais... Washington: IEEE Computer Society, maio 2009. p. 17-24.

FUCHS, C. The internet as a self-organizing socio-technological system. Cybernetics and Human Knowing, Mytilene, v. 12, n. 3, p. 57-81, 2005.

HAYWOOD, Dan. Domain drive design using naked objects: the pragmatic programmers. Lewisville: Pragmatic Bookshelf, 2009.

KILOV, H.; SACK, I. Mechanisms for communication between business and IT experts. Computer. Standards \& Interfaces, Amsterdam, v. 31, n. 1, p. 98-109, jan. 2009.

LAUDON, K. C.; LAUDON, J. P. Sistemas de informação. Rio de Janeiro: LTC, 1999. 
McGEE, J.; PRUSAK, L. Gerenciamento estratégico da informação: aumente a competitividade e a eficácia de sua empresa utilizando a informação como uma ferramenta estratégica. Rio de Janeiro: Campus, 1994.

MING-JI, J. L.; SHIU-WAN, H.; CHIH-JOU, C. Fostering the determinants of knowledge sharing in professional virtual communities. Computers in Human Behavior, Amsterdã, v. 25, n. 4, p. 929-939, jul. 2009.

MORGAN, G. Imagens da organização. São Paulo: Atlas, 1996.

OBJECT MANAGEMENT GROUP. Documents associated with UML version 2.2. fev. 2009. Disponível em: <http://www.omg.org/spec/UML/2.2/>. Acesso em: 3 mar. 2010.

PRESSMAN, R. S. Software engineering: a practitioner approach. 6. ed. Nova York: McGraw Hill, 2005. 908 p. ISBN-007.123840-9.

RIECKEN, R. Frame de temas potenciais de pesquisa em Ciência da Informação. Revista Digital de Biblioteconomia e Ciência da Informação, Brasília, v. 3, n. 2, p. 43-63, mar. 2006.

SARACEVIC, T. Ciência da Informação: origem, evolução e relações. Perspectivas em Ciência da Informação, Belo Horizonte, v. 1, n. 1, p. 41-62, jan./jun. 1996.

SAYAO, L. F. Modelos teóricos em ciência da informação: abstração e método científico. Ciência da Informação, Brasília, v. 30, n. 1, p. 82-91, jan./abr. 2001.

SAWYER, Steve; CROWSTON, Kevin. Information systems in organizations and society: Speculating on the next 25 years of research. In: Information systems research. Boston: Springer, 2004. p. 35-52.

SCHNEIDER, K. Experience and knowledge management in software engineering. Hannover: Springer Verlang Berlin, 2009. 247 p. ISBN 978-3-540-95879-6.

SCHREIBER, G. et al. Knowledge engineering and management: the CommonKADS methodology. Massachusetts: MIT Press, 2000. 471 p.

SHARRATT, M.; USORO, A. Understanding knowledge-sharing in online communities of practice. Electronic Journal on Knowledge Management, Berkshite, v. 1, n. 2, p.187-196, dez. 2003.

SHULLL, F. et al. Knowledge-Sharing issues in experimental software engineering empirical software engineering. Kluwer Academic Publishers, Hingham, v. 9, n. 1, p. 111-137, mar. 2004.

SOMMERVILLE, I. Ingenieria del software. 7.ed. Madrid: Pearson Educacion Sa, 2005. 691 p. ISBN:84-7829-074-5.

WANG, S.; NOE, R. A. Knowledge sharing: a review and directions for future research. Human Resource Management Review, v. 20, n. 2, p. 115-131, jun. 2010.

\section{ABSTRACT}


Requirements engineering, the initial stage in the building of information systems, requires intense exchange of information among professionals of information and communication technologies and business experts. The current theory and practice of requirements engineering, however, are technocentric and characterized by blocked communication. Recent literature discusses the idea of sociotechnological systems, whose properties emerge through the dynamic collaboration among individuals and artificial agents. This article presents a systemic view of requirements engineering based on Mario Bunge's CESM model, under which any concrete system can be represented according to its composition, environment, structure, and mechanism. The composition includes people, artificial agents, and the objects of the dynamic collaboration - domain model and requirements. The mechanism is essentially a communication process, with information exchange and knowledge sharing. This description lends itself to the systemic understanding of requirements engineering aimed at the intervention to alleviate communication problems and to promote the emergence of requirements that represent the system's real necessities.

KEYWORDS: Information systems. Sociotechnological systems. Requirements engineering. Knowledge sharing. Systemic modeling.

Originais recebidos em: 28/03/2010

Aceito para publicação em: 30/08/2010 